\title{
Front Matter: Volume 10624
}

, "Front Matter: Volume 10624," Proc. SPIE 10624, Infrared Technology and Applications XLIV, 1062401 (17 July 2018); doi: 10.1117/12.2320354

SPIE. Event: SPIE Defense + Security, 2018, Orlando, FL, United States 


\title{
PROCEEDINGS OF SPIE
}

\section{Infrared Technology and Applications XLIV}

\author{
Bjørn F. Andresen \\ Gabor F. Fulop \\ Charles M. Hanson \\ John Lester Miller \\ Paul R. Norton \\ Editors
}

16-19 April 2018

Orlando, Florida, United States

Sponsored and Published by

SPIE

Volume 10624 
The papers in this volume were part of the technical conference cited on the cover and title page. Papers were selected and subject to review by the editors and conference program committee. Some conference presentations may not be available for publication. Additional papers and presentation recordings may be available online in the SPIE Digital Library at SPIEDigitalLibrary.org.

The papers reflect the work and thoughts of the authors and are published herein as submitted. The publisher is not responsible for the validity of the information or for any outcomes resulting from reliance thereon.

Please use the following format to cite material from these proceedings:

Author(s), "Title of Paper," in Infrared Technology and Applications XLIV, edited by Bjørn F. Andresen, Gabor F. Fulop, Charles M. Hanson, John Lester Miller, Paul R. Norton, Proceedings of SPIE Vol. 10624 (SPIE, Bellingham, WA, 2018) Seven-digit Article CID Number.

ISSN: 0277-786X

ISSN: 1996-756X (electronic)

ISBN: 9781510617599

ISBN: 9781510617605 (electronic)

Published by

SPIE

P.O. Box 10, Bellingham, Washington 98227-0010 USA

Telephone +1 3606763290 (Pacific Time) · Fax +1 3606471445

SPIE.org

Copyright (C) 2018, Society of Photo-Optical Instrumentation Engineers.

Copying of material in this book for internal or personal use, or for the internal or personal use of specific clients, beyond the fair use provisions granted by the U.S. Copyright Law is authorized by SPIE subject to payment of copying fees. The Transactional Reporting Service base fee for this volume is $\$ 18.00$ per article (or portion thereof), which should be paid directly to the Copyright Clearance Center (CCC), 222 Rosewood Drive, Danvers, MA 01923. Payment may also be made electronically through CCC Online at copyright.com. Other copying for republication, resale, advertising or promotion, or any form of systematic or multiple reproduction of any material in this book is prohibited except with permission in writing from the publisher. The CCC fee code is 0277$786 \mathrm{X} / 18 / \$ 18.00$.

Printed in the United States of America.

Publication of record for individual papers is online in the SPIE Digital Library.

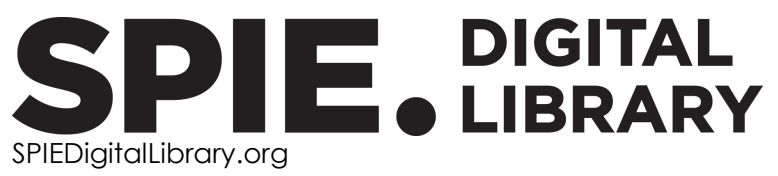

Paper Numbering: Proceedings of SPIE follow an e-First publication model. A unique citation identifier (CID) number is assigned to each article at the time of publication. Utilization of CIDs allows articles to be fully citable as soon as they are published online, and connects the same identifier to all online and print versions of the publication. SPIE uses a seven-digit CID article numbering system structured as follows:

- The first five digits correspond to the SPIE volume number.

- The last two digits indicate publication order within the volume using a Base 36 numbering system employing both numerals and letters. These two-number sets start with 00, 01, 02, 03, 04, 05, 06, 07, 08, 09, 0A, OB ... 0Z, followed by 10-1Z, 20-2Z, etc. The CID Number appears on each page of the manuscript. 


\title{
Contents
}

\author{
ix Authors \\ xiii Conference Committee
}

NIR/SWIR

1062402 Thermoelectrically cooled extended-SWIR FPAs using unipolar barrier detectors (Invited Paper) [10624-1]

$1062403 \quad$ Extended SWIR imaging for targeting and reconnaissance [10624-2]

$1062404 \quad$ High resolution 1.3 megapixel extended wavelength InGaAs (Invited Paper) [10624-3]

1062405 Shockley-Read-Hall (SRH) recombination dark current in planar diffused $\mathrm{P}^{+} \mathrm{n}$ heterostructure $\operatorname{InP} / \mathrm{In}_{0.53} \mathrm{Ga}_{0.47} \mathrm{As} / \mathrm{InP}$ high density small pitch Focal Plane Arrays (FPAs) (Invited Paper) [10624-4]

1062406 Low SWaP SWIR video engine for image intensifier replacement [10624-5]

$1062407 \quad$ Numerical and analytical modeling of bulk and surface generation recombination currents in InGaAs/InP SWIR photodiodes [10624-6]

1062408 Highly efficient MIM diodes for NIR and SWIR applications [10624-7]

1062409 Colorimetry and multispectral imaging in the shortwave infrared [10624-8]

\section{SELECT APPLICATIONS}

10624 OA A holistic approach to high performance infrared system design (Invited Paper) [10624-9]

10624 OB Towards ultra-small pixel pitch cooled MW and LW IR-modules (Invited Paper) [10624-10]

10624 OD Wide field of view visible and near infrared pushbroom airborne hyperspectral imager [10624-12]

10624 OE Design and test results of compact imaging spectrometer for chemical detection and identification [10624-13]

10624 OF Towards a compact, portable, handheld device for contactless real-time standoff detection of hazardous substances [10624-14] 
10624 OG Evaluation of space radiation effects on HgCdTe avalanche photodiode arrays for Lidar applications [10624-15]

T2SL I

10624 Ol Very long wavelength type-II InAs/GaSb superlattice infrared detectors [10624-16]

10624 OJ Type II superlattice infrared photodetector research at Fraunhofer IAF [10624-18]

$10624 \mathrm{OL} \quad$ Transitioning large-diameter Type II Superlattice detector wafers to manufacturing [10624-20]

10624 OM Carrier concentration and transport in Be-doped InAsSb for infrared sensing applications [10624-21]

10624 ON Vertical transport study of InAs/GaSb type-Il superlattice nBp MWIR detectors using electron beam-induced current measurement [10624-22]

1062400 Dual-band MWIR/LWIR focal plane arrays based on III-V strained-layer superlattices [10624-23]

HGCDTE

10624 OR Dark current characterization of Au and Hg-vacancy hybrid doped p-type epitaxy longwavelength $\mathrm{HgCdTe}$ infrared photodetectors [10624-27]

10624 OS High-performance SWIR/MWIR and MWIR/MWIR bispectral MCT detectors by AIM [10624-28]

10624 OT Numerical simulation of passivated long-wave IR HgCdTe surfaces and their effect on detector performance [10624-29]

10624 OU Achievement of high image quality MCT sensors with Sofradir vertical industrial model [10624-31]

10624 OV The status of MCT detector development at ASELSAN [10624-32]

10624 OW From CdZnTe bulk growth to HgCdTe infra-red detectors: mastering the chain for highperformance and reliable imaging [10624-33]

10624 OX Bulk characterization and surface analysis of epitaxy ready cadmium zinc telluride substrates for use in IRFPA manufacturing for IR imaging [10624-34] 
HOT

10624 OY Influence of radiative recombination on performance of p-i-n HOT long wavelength infrared HgCdTe photodiodes (Invited Paper) [10624-35]

$106240 Z$ HOT MWIR detectors on silicon substrates (Invited Paper) [10624-36]

1062410 Antimonide e-SWIR, MWIR, and LWIR barrier infrared detector and focal plane array development [10624-37]

1062411 Potential and limitations for very-high-operating-temperature (VHOT) MWIR focal plane arrays using halogen-passivated PbSe [10624-38]

1062412 HOT MWIR InAs/InAsSb T2SL discrete photodetector development [10624-40]

UNCOOLED FPAS AND APPLICATIONS

1062414 Wide-angle and polarization-selective plasmonic nano-metagrating absorbers [10624-43]

1062415 Broadband photoresponse of graphene photodetector from visible to long-wavelength infrared wavelengths [10624-44]

1062416 Wide-band circular polarized cross bowtie antenna in LWIR [10624-45]

1062417 Single layer microbolometer detector pixel using ZnO material [10624-46]

1062418 Infrared leaky-wave antenna using a uniaxial graphene metasurface [10624-47]

1062419 High-performance uncooled digital $17 \mu \mathrm{m}$ QVGA-IRFPA-using microbolometer based on amorphous silicon with massively parallel Sigma-Delta-ADC readout [10624-48]

10624 IA Physical device modeling of $\mathrm{Si} / \mathrm{Si}_{1-x} \mathrm{Ge}_{\mathbf{x}}$ multi-quantum well detector to optimize Ge content for higher thermal sensitivity [10624-49]

10624 IC Implications of $1 / \mathrm{f}$ noise in uncooled thermal imaging [10624-51]

10624 ID Small uncooled bolometers with a broad spectral response [10624-52]

$106241 \mathrm{E}$ Pixel resistance optimization of a Si..5 Ge.5/Si MQWs thermistor based on in-situ B doping for microbolometer applications [10624-53]

$10624 \mathrm{lF}$ Development of titanium oxide based $12 \mu \mathrm{m}$ pixel pitch uncooled infrared detector [10624-54]

10624 IG Smart digital fusion between visible color and infrared sensors [10624-57] 


\section{SMART PROCESSING}

$10624 \mathrm{1H} \quad$ Spatial and spectral filtering on focal plane arrays [10624-55]

$10624 \mathrm{lJ} \quad$ Small infrared target detection by data-driven proposal and deep learning-based classification [10624-58]

$106241 \mathrm{~L} \quad$ Thermal image improvement methods and their real time implementations on FPGAs [10624-59]

$10624 \mathrm{IM} \quad$ A $1024 \times 512$ ROIC with $30 \mu \mathrm{m}$ pixel pitch and $250 \mathrm{~Hz}$ high frame rate for shortwave infrared detector [10624-60]

QWIP

10624 iN Small pitch resonator-QWIP detectors and arrays [10624-61]

$1062410 \quad$ High-resolution QWIP and T2SL IDDCAs by IRnova [10624-62]

Q-DOTS

$106241 Q \quad$ Short wave infrared photodetector using p-i-p quantum dots (InAs/GaAs) for high temperature operation [10624-64]

10624 IR Modelling of dark current and noise dependence on capping thickness in quantum dots based infrared photodetectors [10624-65]

10624 is Heterogeneously coupled InAs Stranski-Krastanov and submonolayer quantum dot infrared photodetector for next-generation IR imaging [10624-66]

\section{POSTER SESSION}

10624 1T Dark current reduction of InGaAs photodiode by low stress diffusion mask [10624-67]

$106241 \mathrm{U} \quad$ Interface engineering in InSb crystal growth for focal plane array device performance [10624-68]

10624 1X A FEA study on thermal stress of HgCdTe infrared focal plane arrays detector [10624-71]

$106241 Z$ A study on ALD ZnS passivation of HgCdTe IRFPAs detectors [10624-72]

$1062421 \quad$ Implementation of SOI diode uncooled IRFPA in TEC-less and shutter-less operation [10624-75] 
1062422 A low-power CMOS readout IC with on-chip column-parallel SAR ADCs for microbolometer applications [10624-77]

1062423 Improvement in NEDT characteristics of InAs/GaAs quantum dot based 320x256 focal plane array implanted with hydrogen ions [10624-78] 
Proc. of SPIE Vol. 10624 1062401-8

Downloaded From: https://www.spiedigitallibrary.org/conference-proceedings-of-spie on 26 Apr 2023 Terms of Use: https://www.spiedigitallibrary.org/terms-of-use 


\section{Authors}

Numbers in the index correspond to the last two digits of the seven-digit citation identifier (CID) article numbering system used in Proceedings of SPIE. The first five digits reflect the volume number. Base 36 numbering is employed for the last two digits and indicates the order of articles within the volume. Numbers start with 00, 01, 02, 03, 04, 05, 06, 07, 08, 09, OA, OB...0Z, followed by 10-1Z, 20-2Z, etc.

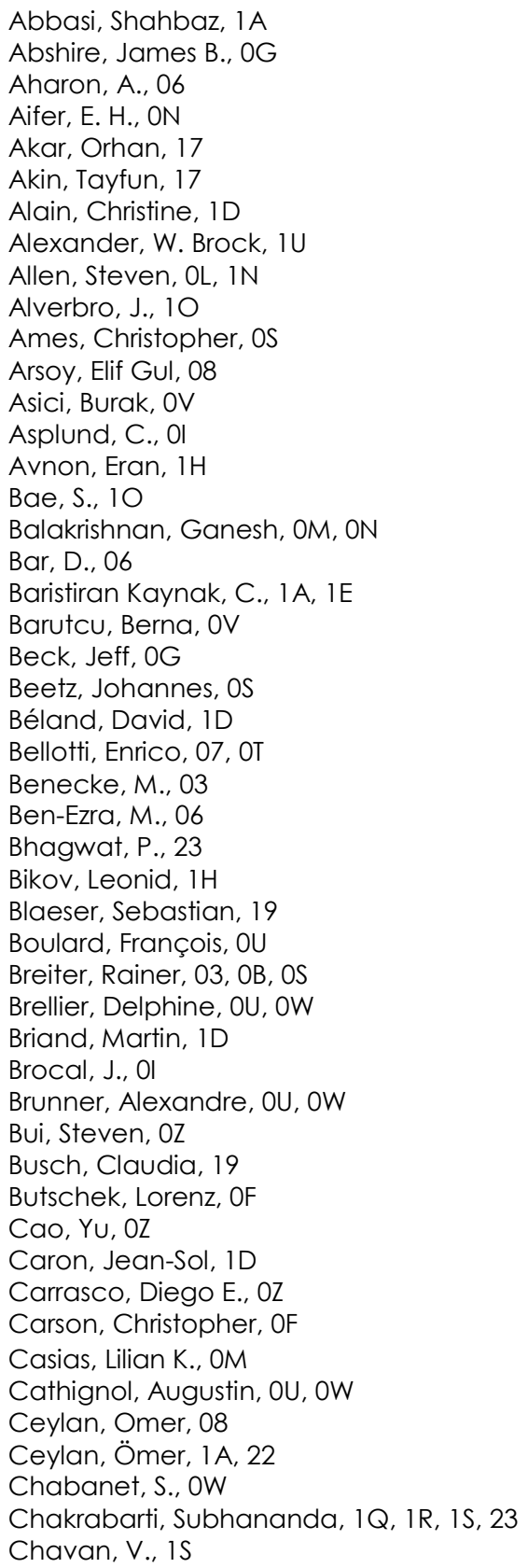

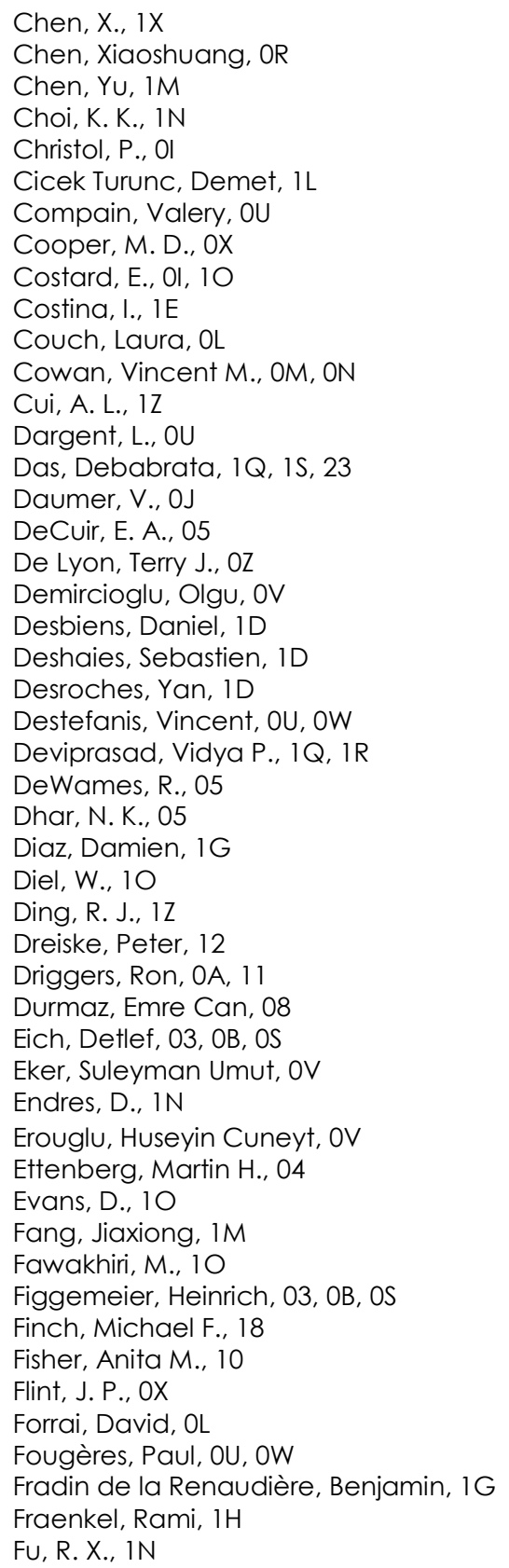


Fujisawa, D., 21

Fukushima, Shoichiro, 15

Garter, Michael, OL

Gay, David, 1D

Gazit, R., 06

Généreux, Francis, 1D

Gerken, M., 09

Geruschke, Thomas, 19

Ghadi, Hemant, 1Q, 1R

Giladi, Avihoo, $1 \mathrm{H}$

Giraud, S., OW

Glasmann, Andreu, 07, 0T

Gliksman, Sivan, $1 \mathrm{H}$

Golding, Terry, 02

Gonzalez, Pilar, 1H

Göritz, A., 1E

Gout, E., OW

Grahmann, Jan, OF

Gramich, V., OJ

Gravrand, Olivier, OU

Gray, Nathan W., $1 \mathrm{U}$

Grimberg, llana, 1H

Guinedor, Pierre, OU

Gunapala, Sarath D., 10

Gunay, Omer, $1 \mathrm{~L}$

Gurbuz, Yasar, 08, 1A, 1E, 22

Gurga, Alexander R., 00

$\mathrm{Ha}$, Chang Soo, $1 \mathrm{~T}$

Haertelt, Marko, OF

Hanna, Stefan, OB, OS

Hanson, Charles M., IC

Hata, H., 21

He, L., 12

Hill, Cory J., 10

Hirsh, Itay, 06, $1 \mathrm{H}$

Hochschulz, Frank, 19

Höglund, L., Ol, 10

Hood, Andrew, 02

Hu, Weida, OR

Hu, X. N., 1 Z

Huang, Edward K., 02

Huang, Songlei, $1 M$

Huang, Zhangcheng, $1 \mathrm{M}$

Hubbs, John E., OG

Hugger, Stefan, OF

Hugger, T., OJ

Ihle, T., 03

Ikhlassi, Amal, 12

Iosevich, R., 06

Ivanov, R., $\mathrm{Ol}$

Jacobsohn, Eli, $1 \mathrm{H}$

Jarvis, Jan-Philip, OF

Jenkins, James R., $\mathrm{OZ}$

Jeong, Yong Jin, IF

Jones, Robert, OL

Jóźwikowski, K., OY

Jung, Han, 1F, IT

Kaldirim, Melih, OV

Kanai, Yasushi, 15

Kang, Sang GU, IF
Kapferer, Armelle, OU, OW

Karni, Yoram, $1 \mathrm{H}$

Kaynak, Mehmet, 1A, 1E, 22

Keo, Sam A., 10

Kerlain, Alexandre, oU

Kim, Jin K., OM

Kim, Jongwoo, 12

Kim, Sungho, $1 \mathrm{~J}$

Kim, Young Ho, $1 \mathrm{~T}$

Kimata, Masafumi, 14

Kimchi, Joe, 12

Kirste, L., OJ

Klinger, $V_{\text {., }}$ OJ

Ko, Sung Yong, $1 T$

Kohn, N., OJ

Kondrashov, P., 06

Kopytko, M., OY

Korndörfera, F., $1 \mathrm{E}$

Kosasayama, Y., 21

Khoshakhlagh, Arezour, 10

Krasovitski, Leonid, 1H

Krishna, Sanjay, OM, ON

Kulse, P., 1E

Kumar, F. J., OX

Kwon, Myung Ho, $1 \mathrm{~F}$

Lail, Brian A., 16, 18

Lambrechts, Andy, $1 \mathrm{H}$

Lange, Michael, 04

Lavenstein, Jean-Marie, OG

Lee, Byoung Wook, $1 T$

Lei, JihFen, 12

Lelievre, Sylviane, $\mathrm{OE}$

Lerch, Renee, 19

Li, Qing, OR

Li, Ray, OZ

Lin, Chun, OR

Littlejohn, D., OA

Liu, L. F., $1 \mathrm{Z}$

Loreau, Y., OW

Louzon, E., 06

Lu, Wei, OR

Lukomsky, Inna, 1H

Luong, Edward M., 10

Luppold, W., OJ

Lutz, H., OB

Macarthur, John, OF

MacDougal, Michael, 02

Mackenzie, J., OX

Mahlein, Karl-Martin, OS

Manzato, M.C., OW

Marcks von Würtemberg, R., Ol

Martin, Christopher R., 04

Martyniuk, P., OY

Mathews, S., ON

Matsumoto, Kazuhiko, 15

Maximenko, S. I., ON

Mediouni, A., 06

Merrell, Jason, $1 \mathrm{U}$

Merten, Andre, OF

Miao, Shenjie, 16 
Michel, Marvin D., 19

Mor, E., 06

Morath, Christian P., OM, ON

Moreau, Louis, OE

Müller, R., OJ

Naureen, S., 0l, 10

Navon, A., 06

Nguyen, Binh-Minh, $\mathrm{OZ}$

Nguyen, Hai, 04

Nicholas, Robert, 11

Niemasz, J., 0J

Nitzani, Michal, $1 \mathrm{H}$

Nosho, Brett Z., 0O, $0 \mathrm{Z}$

Oelmaier, R., OB

Ogawa, Shinpei, 14, 15

Okuda, Satoshi, 15

Okyay, Ali K., 17

Olver, K. A., $1 \mathrm{~N}$

Ono, Takao, 15

Oriot, David, $1 \mathrm{G}$

Ostendorf, Ralf, OF

Ozcan, Meric, 08

Ozer, Selcuk, OV

Ozsarac, Ismail, $1 \mathrm{~L}$

Panda, Debiprasad, 1Q, 1S, 23

Paultre, Jacques-Edmond, ID

Pepper, Brian J., 10

Perron, Gaetan, OE

Petermann, Martin, 19

Poirier, Michel, 1D

Pozzi, M., 10

Prax, Andrew, $1 \mathrm{U}$

Premkumar, Navaneeth, 16, 18

Prigozhin, llya, OT

Provençal, Francis, 1D

Rafol, Sir B., 10

Rahat, $0 ., 06$

Rajavel, Rajesh D., 00, 0 Z

Rangel, Elizabeth, 12

Ratajczyk, Marcin, OF

Rawool, Harshal, 1Q

Regensburger, M., 06

Rehm, R., OJ

Reibel, Y., OW

Rodriguez, J. B., Ol

Rogalski, A., OY

Roman-Tinnes, C., OW

Rossignol, R., Ol

Rouvié, A., 0

Rubaldo, Laurent, OU, OW

Rutz, F., OJ

Ryu, Junhwan, $1 \mathrm{~J}$

Saha, J., is

Sahoo, Swetapadma, IR

Saint-Pé, O., 01

Sam-giao, D., OU

San, Ayse, OV

Sarac, Adem, 17

Sasmaz, Emrah, OV

Satake, T., 21
Savchenko, M., 06

Schallenberg, Timo, OS

Schlemmer, H., 09

Schmidt, J., OJ

Scholten, M., OA

Schulz, K., 1E

Schuster, J., 05

Schwarzenberg, Markus, OF

Shafique, Atia, 1A, 1E, 22

Shay, A., 06

Shelton, David, 11

Shimatani, Masaaki, 15

Shkedy, Lior, $1 \mathrm{H}$

Short, R., OA

Shtrichman, Itay, 06, 1H

Shunam, E., 06

Sieck, Alexander, 03, 0S

Siemens, C., 09

Sigley, Justin, 11

Soibel, Alexander, 10

Soulié, Emmanuel, $1 \mathrm{G}$

Stadelmann, T., OJ

Steenbergen, Elizabeth $\mathrm{H} ., \mathrm{OM}, \mathrm{ON}$

Stein, Matthias, 19

Stothard, David, OF

Subrahmanyam, N.B.V., 23

Sullivan, William, OG

Sun, C. H., 1 Z

Sun, J. G., IN

Sun, Xiaoli, OG

Suzuki, D., 21

Taghipour, Z., ON

Takenaga, T., 21

Takikawa, T., 21

Tanguay, Francois, $\mathrm{OE}$

Tanrikulu, M. Yusuf, 17

Terterian, Sevag, 00

Thomas, Jeremy, 02

Ting, David Z., 10

Tolungüç, Alp, OV

Tremblay, Bruno, 1D

Tuito, A., 06

Upadhyay, S., 23

Vogt, Holger, 19

Vollmerhausen, R., OA

Walther, M., OJ

Wang, Jianyu, OD

Wang, Shengwei, OD

Wang, Shuogin, 00

Wang, Xuquan, $1 \mathrm{M}$

Wang, Yueming, OD

Warden, Matthew, OF

Wauro, M., OJ

Weber, A., 03

Webster, Preston T., OM

Wei, Yajun, OL

Weiler, Dirk, 19

Weinstein, M., 06

Wendler, J., O3, OB

Wenisch, Jan, OS 
Weyers, Sascha, 19

Wicks, Gary, 02

Wietstruck, M., 1E

Williams, Adam J., $\mathrm{OZ}$

Wörl, A., OJ

Würfel, Daniel, 19

$\mathrm{XU}$, Yuancheng, 16

Yamamoto, Y., IE

Yamashita, K., 21

Yazici, Melik, 22

Ye, Z. H., $1 \mathrm{X}, 1 \mathrm{Z}$

Yildizak, Ciğdem, 17

Yu, Yanan, OD

Yuan, Henry, 12

Yuan, Liyin, OD

Zaumseil, P., 1E

Zhang, W. T., IX

Zhao, Ding, OD 


\title{
Conference Committee
}

\author{
Symposium Chair
}

Arthur A. Morrish, Raytheon Space and Airborne Systems

(United States)

Symposium Co-chair

Ruth Moser, Air Force Research Laboratory (United States)

Conference Chairs

Bjørn F. Andresen, Consultant, Infrared Technologies \& Applications (Israel)

Gabor F. Fulop, Maxtech International, Inc. (United States) and Infrared Imaging News (United States)

Charles M. Hanson, SenselR Solutions, LLC (United States) John Lester Miller, Cascade Electro Optics, LLC (United States)

Paul R. Norton, U.S. Army Night Vision \& Electronic Sensors Directorate (United States)

Conference Program Committee

Tayfun Akin, Mikro-Tasarim Ltd. (Turkey) and Middle East Technical University (Turkey)

Sooho Bae, i3system, Inc. (Korea, Republic of)

Eric Belhaire, Thales Optronique S.A.S. (France)

Wolfgang A. Cabanski, AIM INFRAROT-MODULE GmbH (Germany)

John T. Caulfield, Cyan Systems (United States)

Leonard P. Chen, Raytheon Vision Systems (United States)

Eric Costard, IRnova AB (Sweden)

Ronald G. Driggers, St. Johns Optical Systems (United States)

Michael T. Eismann, Air Force Research Laboratory (United States)

Martin H. Ettenberg, Princeton Infrared Technologies, Inc. (United States)

Mark E. Greiner, L3 Cincinnati Electronics (United States)

Sarath D. Gunapala, Jet Propulsion Laboratory (United States)

Weida Hu, Shanghai Institute of Technical Physics (China)

Arjun KarRoy, Jazz Semiconductor, Inc. (United States)

Masafumi Kimata, Ritsumeikan University (Japan)

Hee Chul Lee, KAIST (Korea, Republic of)

Paul D. LeVan, Air Force Research Laboratory (United States)

Kevin C. Liddiard, Electro-optic Sensor Design (Australia) 
Wei Lu, Shanghai Institute of Technical Physics (China)

Michael H. MacDougal, Attollo Engineering, LLC (United States)

Tara J. Martin, UTC Aerospace Systems (United States)

Paul L. McCarley, Air Force Research Laboratory (United States)

R. Kennedy McEwen, Leonardo MW Ltd. (United Kingdom)

A. Fenner Milton, U.S. Army RDECOM CERDEC NVESD (United States)

Mario O. Münzberg, HENSOLDT Optronics GmbH (Germany)

Peter W. Norton, BAE Systems (United States)

Vladimir P. Ponomarenko, Orion Research-and-Production Association (Russian Federation)

Manijeh Razeghi, Northwestern University (United States)

Donald A. Reago Jr., U.S. Army RDECOM CERDEC NVESD (United States)

Colin E. Reese, U.S. Army Night Vision \& Electronic Sensors Directorate (United States)

Patrick Robert, ULIS (France)

Antoni Rogalski, Military University of Technology (Poland)

Laurent Rubaldo, SOFRADIR (France)

Thomas R. Schimert, DRS Technologies, Inc. (United States)

Itay Shtrichman, SCD Semiconductor Devices (Israel)

Torbjørn Skauli, Norwegian Defence Research Establishment (Norway)

Bharadwaja Srowthi, L3 Infrared Products (United States)

Stefan P. Svensson, U.S. Army Research Laboratory (United States)

J. Ralph Teague, Georgia Tech Research Institute (United States)

Simon Thibault, Université Laval (Canada)

Meimei Tidrow, U.S. Army Night Vision \& Electronic Sensors Directorate (United States)

James R. Waterman, U.S. Naval Research Laboratory (United States)

Ami Yaacobi, Rafael Advanced Defense Systems Ltd. (Israel)

Lucy Zheng, Institute for Defense Analyses (United States)

\section{Session Chairs}

$1 \quad \mathrm{NIR} / \mathrm{SWIR}$

Martin H. Ettenberg, Princeton Infrared Technologies, Inc. (United States)

Wolfgang A. Cabanski, AIM INFRAROT-MODULE GmbH (Germany)

2 Select Applications

Weida Hu, Shanghai Institute of Technical Physics (China)

Mario O. Münzberg, HENSOLDT Optronics GmbH (Germany) 
3 T2SLI

Meimei Z. Tidrow, U.S. Army Night Vision \& Electronic Sensors

Directorate (United States)

Lucy Zheng, Institute for Defense Analyses (United States)

Philip C. Klipstein, SCD SemiConductor Devices (Israel)

4 Keynote Session

Gabe F. Fulop, Maxtech International, Inc. (United States)

5 T2SL II

Meimei Z. Tidrow, U.S. Army Night Vision \& Electronic Sensors Directorate (United States)

Lucy Zheng, Institute for Defense Analyses (United States)

Philip C. Klipstein, SCD SemiConductor Devices (Israel)

$6 \quad \mathrm{HgCdTe}$

Whitney Mason, DARPA (United States)

7 HOT

Michael T. Eismann, Air Force Research Laboratory (United States)

Antoni Rogalski, Military University of Technology (Poland)

8 A Word from the Masters

Paul Norton, U.S. Army Night Vision \& Electronic Sensors Directorate (United States)

9 Uncooled FPAs and Applications

Kevin C. Liddiard, Electro-optic Sensor Design (Australia)

Colin E. Reese, U.S. Army Night Vision \& Electronic Sensors Directorate (United States)

Masafumi Kimata, Ritsumeikan University (Japan)

10 Smart Processing

John T. Caulfield, Cyan Systems, Inc. (United States)

Paul L. McCarley, Air Force Research Laboratory (United States)

11 QWIP

Eric M. Costard, IRnova AB (Sweden)

12 Q-Dots

John Lester Miller, Cascade Electro-Optics, LLC (United States) 
Proc. of SPIE Vol. 10624 1062401-16 Downloaded From: https://www.spiedigitallibrary.org/conference-proceedings-of-spie on 26 Apr 2023
Terms of Use: https://www.spiedigitallibrary.org/terms-of-use 